\title{
Current opinion on treatment of inflammatory bowel disease in pregnant women
}

Pooneh Salari', Shekoufeh Nikfar ${ }^{2,3}$, Mohammad Abdollahi $^{4}$

\author{
${ }^{1}$ Medical Ethics and History of Medicine Research Center, Tehran University \\ of Medical Sciences, Tehran, Iran \\ 2Department of Pharmacoeconomics and Pharmaceutical Administration, \\ Faculty of Pharmacy, Tehran University of Medical Sciences, Tehran, Iran \\ ${ }^{3}$ Food and Drug Laboratory Research Center, Food and Drug Organization, \\ Ministry of Health and Medical Education, Tehran, Iran \\ ${ }^{4}$ Faculty of Pharmacy, and Pharmaceutical Sciences Research Center, Tehran University \\ of Medical Sciences, Iran
}

Submitted: 7 September 2012

Accepted: 9 September 2012

Arch Med Sci 2012; 8, 6: 983-986

DOI: 10.5114 /aoms.2012.32403

Copyright (C) 2012 Termedia \& Banach

Inflammatory bowel disease (IBD) refers to a relapsing and remitting disease representing as forms of ulcerative colitis (UC) and Crohn's disease (CD) [1]. The peak age of onset is between 20 and 40 years of age, and thus overlaps with child-bearing years [2]. Fear of the adverse effect of medication on pregnancy is highly established in women with IBD, yet awareness of the harmful effect of IBD relapse during pregnancy is poor [3]. Generally both the active disease and its treatment may affect pregnancy; however, the belief is that the risk of the active disease is always greater than its medications [4].

Active CD and UC during conception and pregnancy increase the risk of adverse prenatal outcomes such as low birth weight and preterm delivery. Therefore active treatment of the disease and establishing remission before conception is the main goal in young women. In a very recent study, Bortoli et al. evaluated pregnancy outcome in IBD patients in a prospective European multicenter case-control study. They demonstrated no significant difference in frequency of fetal abnormalities in IBD patients compared with non-IBD controls [5].

Van der Eoude et al. found that the risk of relapse after conception is the same as non-pregnant IBD patients but if pregnancy occurs during disease flare-up, the disease will remain persistently active during pregnancy [6]. Inflammatory bowel disease itself, especially CD, may increase the risk of adverse neonatal outcome [6]. The risk is not only related to the medications but also related to the disease severity. One of the most important improvements in the management of IBD over the past decade has been the finding that normal pregnancy outcomes can be accomplished when a woman enters pregnancy in remission [7]. New insights into the safety of a wider spectrum of drugs in these patients have a great role in increasing success in IBD management.

Various classes of drugs are used in disease management including aminosalicylates, corticosteroids, immunosuppressive drugs, antibiotics, and biologic agents. Also in recent years, the effectiveness of probiotics in maintaining remission and their efficacy in preventing relapse in IBD have been supported by concrete evidence $[8,9]$.

\author{
Corresponding author: \\ Prof. Mohammad Abdollahi \\ Faculty of Pharmacy \\ and Pharmaceutical Sciences \\ Research Center \\ Tehran University \\ of Medical Sciences \\ Tehran 1417614411, Iran \\ Phone/fax: +98 2166959104 \\ E-mail: mohammad.abdollahi@ \\ utoronto.ca
}


Aminosalicylates - the standard treatment for induction and maintenance of remission of mild to moderate forms of IBD (mostly UC) - are poorly absorbed into blood circulation but once in the blood can easily cross the placenta and reach the fetus [10-12]. They are relatively safe during pregnancy and are considered as class B by the Food and Drug Administration (FDA) but folic acid supplementation at $2 \mathrm{mg}$ per day is recommended to overcome probable risks of folate deficiency and its complications in neonates. In a metaanalysis in 2007, Rahimi et al. reported a 1.16-fold increase in congenital malformations, 2.38-fold increase in stillbirth, 1.14-fold increase in spontaneous abortion, 1.35 -fold increase in preterm delivery, and 0.93 -fold increase in low birth weight in IBD pregnant women on aminosalicylate therapy [13] but they did not compare the risk in IBD pregnant women without drug therapy. A few studies reported the risk of neonatal interstitial nephritis with higher doses of mesalazine (more than $3 \mathrm{~g} /$ day) $[14,15]$.

Corticosteroids are classified as class $C$ drugs in pregnancy. There is no evidence of teratogenicity of corticosteroids and they are prescribed for different forms of the disease [16]. Although there are differences between corticosteroids there is no report of maternal adrenal suppression, glucose intolerance, ocular side effects, hypertension, or congenital abnormalities with budesonide [17], which is commonly used in IBD, whereas an increase in the risk of oral cleft was found with prednisone in a meta-analysis [18]. Of course, the route of drug administration may affect drug plasma levels as well as its placental transfer [16]. Budesonide is mainly used as a rectal enema while prednisolone is used orally.

There is no consensus among practitioners on the adverse fetal consequences after immunosuppressive exposure. The use of mercaptopurine during pregnancy is not recommended because of miscarriage and preterm birth and it is considered as class D. Although some studies could not show adverse pregnancy outcomes with mercaptopurine and azathioprine (AZA) [19, 20], others reported congenital abnormalities [21]. Cleary and Källén indicated a moderate risk of congenital malformations, specifically ventricular/atrial septal defects as well as growth retardation [22], but they did not consider the effect of the disease on pregnancy. In contrast, Shim et al. found no association between AZA/mercaptopurine and risk of preterm birth, fetal adverse outcomes and congenital anomalies in IBD pregnant women [23]. Cyclosporine can be indicated in severe cases unresponsive to steroids because of serious health concerns [24]. There are reports of pros and cons of the association of cyclosporine neonatal exposure and adverse outcome [10]. To date, there are no conclusive data about its safety; nevertheless, its use in pregnancy is not recommended.

Biologic agents are considered as a useful class of drugs in the course of IBD in unresponsive and severe forms. Infliximab, a monoclonal anti-tumor necrosis factor- $\alpha$ antibody, is an effective biologic agent for induction and maintenance of remission of CD and is classified as a class B drug in pregnancy. It crosses the placental barrier at week 20 and thereafter in a linear fashion can be detected in newborns [25]. There are a few reports of infliximab-induced malformations [26] but the results of most of the studies are in favor of infliximab $[27,28]$. Animal studies did not show adverse fetal effects [29]. Adalimumab is also considered as a class $B$ drug for use in pregnancy and no congenital abnormalities were reported $[30,31]$ The safety of certolizumab in pregnancy is not fully known. A recent observational study on the risk/ benefit assessment of anti-TNF treatment with infliximab and adalimumab in 212 pregnant women determined the incidence of adverse outcomes as not higher than for IBD [32].

Zelinkova et al. assessed 4 pregnant IBD patients under infliximab treatment and determined 2-3fold higher infliximab concentrations in the cord blood than peripheral blood of mothers. After stopping infliximab, the pregnant women were followed for 3-6 months and normal neonatal development was observed; however, this study raised a debate on the unknown effects of infliximab on the developing immune system [33].

Biological therapy may cause serious side effects including pneumonia, tuberculosis, lymphoma, drug-induced lupus, and hepatotoxicity in a small percentage of patients, and the balance between their risks and benefits seems to be good [34].

Antibiotics are also used in IBD patients, especially $C D$, on some occasions and even have been even proposed effective in irritable bowel syndrome (IBS) [35]. They include metronidazole, ciprofloxacin, tetracyclines and sulfonamides, which are associated with high risk of neonatal anomalies or contraindicated while ampicillin, cephalosporin and erythromycin are associated with low risk of adverse pregnancy outcomes [36].

Probiotics that are recently approved in management of IBD and even IBS [37] do not appear to cause any safety concerns for pregnant women $[8$, 9]. Systemic absorption is rare when probiotics are used, and the current literature does not indicate any increase in adverse pregnancy outcomes [38].

There is a great fear of adverse medication effects on pregnancy outcomes among IBD patients while there are a few conflicting and controversial studies which reported the adverse effects of IBD medications on pregnancy outcomes. In contrast, 
the knowledge of IBD patients about the deleterious effects of the disease on pregnancy outcome is inadequate while the disease activity carries the highest risk.

\section{Expert opinion}

Taking all the evidence into consideration, the prescribers should consider the risk-benefit assessment of each class of drugs during conception and pregnancy. Defining the best therapeutic approach in pregnancy, different scenarios must be considered in IBD pregnant women including planning pregnancy in patients in remission; unplanned pregnancies in patients with active disease; planning pregnancy in patients with active disease; and unplanned pregnancy in patients in remission. In each of these scenarios, the clinician must evaluate the patient's drug regimen and plan to change some of its components if necessary. It should be noted that drug discontinuation because of the fear of adverse pregnancy outcomes worsens the situation and the mother will be at a high risk of flareup. Generally, aminosalicylates and corticosteroids are routinely used for moderate to severe forms and data about their safety are growing. Although the risk of serious adverse drug reactions during pregnancy is low, during pregnancy methotrexate and thalidomide are contraindicated and after pregnancy if the patient is on treatment with these two drugs, changing medications is necessary. The most important issue regarding anti-TNF agents is their probable side effect on the developing immune system, which needs more investigations, and for this purpose and according to a study there is the possibility of stopping infliximab in the third trimester and resuming drug administration after delivery. Notably, infliximab does not reduce the rate of colectomy in patients with IBD while colectomy and surgical resection with ileal pouch anal anastomosis (IPAA) have an important role in induction of infertility in IBD patients $[39,40]$. In UC, overall fertility rates are normal except after surgery. Therefore considering more effective and safe management to prolong remission and protection of patients to reach the phase of surgery are very important. Most medications in the treatment of IBD are safe for the fetus and should be continued throughout pregnancy in order to maintain maternal health. It should be emphasized that maintaining fertility of patients and safety of pregnancy are dependent on remission of patients. Therefore, more effective and not risky agents such as probiotics could stay in the center of interest.

There are still questions about new medications such as monoclonal antibodies for which data are rare and time is needed to gather case reports. Notably, conducting clinical trials is unethical in pregnant patients and thus researchers should rely on observational studies, which are still rare.

\section{References}

1. Salari-Sharif P, Abdollahi M. Phosphodiesterase 4 inhibitors in inflammatory bowel disease: a comprehensive review. Curr Pharm Des 2010; 16: 3661-7.

2. Moleski SM, Choudhary C. Special considerations for women with IBD. Gastroenterol Clin N Am 2011; 40: 387-98.

3. Mountifield RE, Prosser R, Bampton P, Muller K, Andrews JM. Pregnancy and IBD treatment: this challenging interplay from a patients' perspective. J Crohns Colitis 2010; 4: 176-82.

4. Moscandrew M, Kane S. Inflammatory bowel diseases and management considerations: fertility and pregnancy. Curr Gastroenterol Rep 2009; 11: 395-9.

5. Bortoli A, Pedersen N, Duricova D, et al. Pregnancy outcome in inflammatory bowel disease: prospective European case-control ECCO-EpiCom study, 2003-2006. Alim Pharmacol Ther 2011; 34: 724-34.

6. van der Woude CJ, Kolacek S, Dotan I, et al. European evidenced-based consensus on reproduction in inflammatory bowel disease. J Crohns Colitis 2010; 4: 493-510.

7. Vermeire S, Carbonnel F, Coulie PG, et al. Management of inflammatory bowel disease in pregnancy. J Crohns Colitis 2012; 6: 811-23.

8. Rahimi R, Nikfar S, Rahimi F, et al. A meta-analysis on the efficacy of probiotics for maintenance of remission and prevention of clinical and endoscopic relapse in Crohn's disease. Dig Dis Sci 2008; 53: 2524-31.

9. Rahimi R, Nikfar S, Rezaie A, Abdollahi M. A meta-analysis of the benefit of probiotics in maintaining remission of human ulcerative colitis: Evidence for prevention of disease relapse and maintenance of remission. Arch Med Sci 2008; 4: 185-90.

10. Cassina M, Fabris L, Okolicsanyi L, et al. Therapy of inflammatory bowel diseases in pregnancy and lactation. Expert Opin Drug Saf 2009; 8: 695-707.

11. Rahimi R, Nikfar S, Rezaie A, Abdollahi M. Comparison of mesalazine and balsalazide in induction and maintenance of remission in patients with ulcerative colitis: a meta-analysis. Dig Dis Sci 2009; 54: 712-21.

12. Nikfar S, Rahimi R, Rezaie A, Abdollahi M. A meta-analysis of the efficacy of sulfasalazine in comparison with 5 -aminosalicylates in the induction of improvement and maintenance of remission in patients with ulcerative colitis. Dig Dis Sci 2009; 54: 1157-70.

13. Rahimi R, Nikfar S, Rezaie A, Abdollahi M. Pregnancy outcome in women with inflammatory bowel disease following exposure to 5 -aminosalicylic acid drugs: a metaanalysis. Reprod Toxicol 2008; 25: 271-5.

14. Marteau P, Tennenbaum R, Elefant E, Lémann M, Cosnes J. Foetal outcome in women with IBD treated during pregnancy with oral mesalazine microgrannules. Alim Pharmacol Ther 1998; 12: 1101-8.

15. Diav-Citrin O, Park YH, Veerasuntharam G, et al. The safety of mesalazine in human pregnancy: a prospective controlled cohort study. Gastroenterology 1998; 114: 23-8.

16. Correia LM, Bonilha DQ, Ramos JD, Ambrogini O, Miszputen SJ. Treatment of inflammatory bowel disease and pregnancy: a review of the literature. Arq Gastroenterol 2010; 47: 197-201.

17. Beaulieu DB, Ananthakrishnan AN, Issa $M$, et al. Budesonide induction and maintenance therapy for Crohns disease during pregnancy. Inflamm Bowel Dis 2009; 15: 25-8.

18. Park-Wyllie L, Mazzotta P, Pastuszak A, et al. Birth defects after maternal exposure to corticosteroids: prospective 
cohort study and meta-analysis of epidemiological studies. Teratology 2000; 62: 385-92.

19. Francella A, Dyan A, Bodian C, Rubin P, Chapman M, Present $\mathrm{DH}$. The safety of 6 -mercaptopurine for childbearing patients with inflammatory bowel disease: a retrospective cohort study. Gastroenterology 2003; 124: 9-17.

20. Moskovitz DN, Bodian C, Chapman ML, et al. The effect on the fetus of medications used to treat pregnant inflammatory bowel-disease patients. Am J Gastroenterol 2004; 99: 656-61.

21. Nørgård B, Pdersen L, Christensen LA, Sørensen HT. Therapeutic drug use in women with Crohns disease and birth outcomes: a Danish nationwide cohort study. Am J Gastroenterol 2007; 102: 1406-13.

22. Cleary BJ, Källén B. Early pregnancy azathiopurine use and pregnancy outcomes. Birth Defects Res 2009; 85: 647-54.

23. Shim L, Eslick GD, Simring AA, Murray H, Weltman MD. The effects of azathiopurine on birth outcomes in women with inflammatory owel disease (IBD). J Crohn Colitis 2011; 5: 234-8.

24. Dozois EJ, Wolff BG, Tremaine WJ, et al. Maternal and fetal outcome after colectomy for fulminant ulcerative colitis during pregnancy: case series and literature review. Dis Colon Rectum 2006; 49: 64-73.

25. Kane SV, Acquah LA. Placental transport of immunoglobulins: a clinical review for gastroenterologists who prescribe therapeutic monoclonal antibodies to women during conception and pregnancy. Am J Gastroenterol 2009; 104: 228-33.

26. Srinivasan R. Infliximab treatment and pregnancy outcome in active Crohns disease. Am J Gastroenterol 2001; 96: 2274-75.

27. O’Donnell S, O'Morain C. Use of antitumor necrosis factor therapy in inflammatory bowel disease during pregnancy and conception. Alim Pharmacol Ther 2008; 27: 885-94.

28. Mahadevan U, Kane S, Sandborn WJ, et al. Intentional infliximab use during pregnancy for induction or maintenance of remission in Crohns disease. Aliment Pharmacol Ther 2005; 21: 733-8.

29. Ferrero S, Ragni N. Inflammatory bowel disease: management issues during pregnancy. Arch Gynecol Obstet 2004; 270: 79-85.

30. Subhani JM, Hamiliton MI. The management of inflammatory bowel disease during pregnancy. Alim Pharmacol Ther 1998; 12: 1039-53.

31. Mishkin DS, Van Deinse W, Becker JM, Farraye FA. Successful use of adalimumab (Humira) for Crohns disease in pregnancy. Inflamm Boel Dis 2006; 12: 827-8.

32. Schnitzler F, Fidder H, Ferrante $M$, et al. Outcome of pregnancy in women with inflammatory bowel disease treated with antitumor necrosis factor therapy. Inflamm Bowel Dis 2011; 17: 1846-54.

33. Zelinkova Z, de Haar C, de Ridder L, et al. High intrauterine exposure to infliximab following maternal antiTNF treatment during pregnancy. Aliment Pharmacol Ther 2011; 33: 1053-8.

34. Van Assache G, Lewis JD, Lichtenstein GR, et al. The London Position Statement of the World Congress of Gastroenterology on Biological Therapy for IBD With the European Crohns and Colitis Organization: safety. Am J Gastroenterol 2011; 106: 1594-602.

35. Rezaie A, Nikfar S, Abdollahi M. The place of antibiotics in management of irritable bowel syndrome: a systematic review and meta-analysis. Arch Med Sci 2010; 6: 49-55.

36. Beaulieu DB, Kane S. Inflammatory bowel disease in pregnancy. World J Gastroenterol 2011; 17: 2696-701.
37. Hosseini A, Nikfar S, Abdollahi M. Are probiotics effective in management of irritable bowel syndrome? Arch Med Sci 2012; 8: 403-5.

38. Elias J, Bozzo P, Einarson A. Are probiotics safe for use during pregnancy and lactation? Can Fam Physician 2011; 57: 299-301.

39. Dubinsky M, Abraham B, Mahadevan U. Management of the pregnant IBD patient. Inflamm Bowel Dis 2008; 14 1736-50.

40. Ehteshami-Afshar S, Nikfar S, Rezaie A, Abdollahi M. A systematic review and meta-analysis of the effects of infliximab on the rate of colectomy and post-operative complications in patients with inflammatory bowe disease. Arch Med Sci 2011; 7: 1000-12. 